\title{
Theory of Vibrations in Stewart Platforms
}

\author{
J.M. Selig and X. Ding* \\ School of Computing, Info. Sys. \& Maths. \\ South Bank University \\ London SE1 0AA, U.K. \\ (seligjm@sbu.ac.uk)
}

\begin{abstract}
This article develops a simple linear model for the motion of a Stewart platform in a stationary position. That is, the situation where the platform is at rest and is then subject to an impulsive disturbance. The hydraulic actuators are modelled as simple spring-dashpot systems and the stiffness and damping matrices of the system are derived. It is found that the damping and stiffness matrices are simply proportional to each other and this simplifies the dynamics greatly. The general solution to the equations of motion is a linear combination of eigensolutions. The eigensolutions are damped oscillations about the harmonic screws of the undamped system. The characteristic values associated with each eigensolution can also be found in terms of the undamped frequencies. Finally some remarks are made concerning the possibility of the system being fully damped.
\end{abstract}

\section{Introduction}

Consider a parallel manipulator such as a Stewart platform. Suppose that the manipulator is stationary and receives an impulsive wrench. How will the manipulator behave? In this work a simple model of the manipulator is discussed to answer this question. The simple model treats the legs of the robots as unstretched springs. If the robot's actuators are hydraulic rams then this seems a reasonable first step. Usually the hydraulic fluid is considered incompressible but this cannot be strictly true. Moreover, to first order no bending will occur in the legs because of the passive spherical joints

* permanent address: Robotics Research Institute, Beijing University of Aero.\& Astro., P. R. China at the ends of each leg. The stiffness of the solid part of the legs will be much greater that the effective stiffness of the hydraulic fluid so the compression of the fluid will provide the dominant restoring force. We expect the stiffness to be high but not infinite.

Account must also be taken of the damping in the hydraulic actuators. A simple viscous model will be assumed. Hence, it seems reasonable to model each leg as simple spring and dashpot.

To introduce the notation used, the next section will briefly review the equations of motion for a rigid body using screw theory.

\section{Notation}

The Newton-Euler equations of motion for a rigid body, can be written very compactly in terms of screws as,

$$
N \ddot{\mathbf{s}}+\{\dot{\mathbf{s}}, N \dot{\mathbf{s}}\}=\mathcal{W} .
$$

Here $\dot{\mathbf{s}}$ is the velocity screw of the rigid body that is,

$$
\dot{\mathbf{s}}=\left(\begin{array}{l}
\omega \\
\mathbf{v}
\end{array}\right)
$$

with $\boldsymbol{\omega}$ and $\mathbf{v}$ the angular and linear velocities of the body. The velocity of an arbitrary point $\mathbf{r}$ on the body is given by the simple relation,

$$
\dot{\mathbf{r}}=\boldsymbol{\omega} \times \mathbf{r}+\mathbf{v}
$$

The time derivative of this screw is another screw $\ddot{\mathbf{s}}$, it is tempting to call this the acceleration screw of the rigid body but it should be remembered that it is not related to the 
acceleration of points in the body in the same way that that the velocities are. In fact, we have the following relation for the acceleration of points in the body,

$$
\ddot{\mathbf{r}}=\dot{\boldsymbol{\omega}} \times \mathbf{r}+\dot{\mathbf{v}}+\boldsymbol{\omega} \times(\boldsymbol{\omega} \times \mathbf{r})+\boldsymbol{\omega} \times \mathbf{v}
$$

The applied wrench $\mathcal{W}$ is not a screw but an element of the vector space dual to the screws, that is an element of the dual to the Lie algebra. The wrench combines the force and moment acting on the body, in partitioned form this can be written,

$$
\mathcal{W}=\left(\begin{array}{c}
\mathbf{M} \\
\mathbf{F}
\end{array}\right)
$$

where $\mathbf{M}$ and $\mathbf{F}$ are the moment vector and force vector respectively. The product $\mathcal{W}^{T}$ s gives a scalar, namely the work done when the body, subject to the wrench $\mathcal{W}$ moves through a small displacement given by the screw $\mathbf{s}$.

A screw can be transformed into a wrench by multiplying it by the $6 \times 6$ inertia matrix of the body $N$. In partitioned form this matrix has the form,

$$
N=\left(\begin{array}{cc}
\mathbf{I} & m C \\
m C^{T} & m I_{3}
\end{array}\right)
$$

where $\mathbf{I}$ is the usual $3 \times 3$ inertia matrix of the body, $m$ is the mass of the body, $I_{3}$ the $3 \times 3$ identity matrix and $C$ the anti-symmetric matrix corresponding to the position of the body's centre of mass $\mathbf{c}$, that is $C \mathbf{x}=\mathbf{c} \times \mathbf{x}$ for any vector $\mathbf{x}$. This construction can be used to give the kinetic energy of the rigid body as,

$$
E_{k}=\frac{1}{2} \dot{\mathbf{s}}^{T} N \dot{\mathbf{s}}
$$

The coriolis terms in the equation of motion above are given by the bracket, $\{\dot{\mathbf{s}}, N \dot{\mathbf{s}}\}$. This a pairing between a screw and a wrench, the result is another wrench. In partitioned form this can be written as,

$$
\{\mathbf{s}, \mathcal{W}\}=\left\{\left(\begin{array}{c}
\boldsymbol{\omega} \\
\mathbf{v}
\end{array}\right),\left(\begin{array}{c}
\mathbf{M} \\
\mathbf{F}
\end{array}\right)\right\}=\left(\begin{array}{c}
\boldsymbol{\omega} \times \mathbf{M}+\mathbf{v} \times \mathbf{F} \\
\boldsymbol{\omega} \times \mathbf{F}
\end{array}\right)
$$

For more detail on the above see [6]. In the following sections we will look at the applied wrench $\mathcal{W}$ and find the contributions caused by the stiffness and damping of the hydraulic actuators.

\section{Stiffness Matrix}

The equation of motion given in the previous section can be applied directly to the moving platform of a parallel manipulator such as the Stewart platform. Assume that the platform is stationary and is then subject to a impulsive wrench. If the displacement of the platform is small we may represent it by a screw $\mathbf{z}$. Further, we will assume that the velocities are small so that the coriolis term, which are quadratic in the velocities, can be neglected. Now the wrench due to a small displacement can be written in terms of the stiffness matrix of the system,

$$
\mathcal{W}_{s}=K \mathbf{z}
$$

Moreover, for a parallel system like a Stewart manipulator, it is well known that the stiffness matrix of the legs sum to give the stiffness matrix of the system.

The stiffness of a single leg can be modelled by an unstretched spring. The spring will be aligned along the leg so that any forces produced will be in the direction determined by the centres of the passive joints at either end of the leg, see fig. 1. The stiffness matrix of such a spring is well known be,

$$
K_{i}=k \mathbf{w}_{i} \mathbf{w}_{i}^{T}
$$

see [3] for example. Here $k$ is the stiffness constant of the spring and $\mathbf{w}_{i}$ is the unit wrench in the direction of the spring,

$$
\mathbf{w}_{i}=\frac{1}{\left|\mathbf{b}_{i}-\mathbf{a}_{i}\right|}\left(\begin{array}{c}
\mathbf{a}_{i} \times \mathbf{b}_{i} \\
\mathbf{b}_{i}-\mathbf{a}_{i}
\end{array}\right)
$$

The centres of the passive spherical joints on the base and moving platform have been written as $\mathbf{a}_{i}$ and $\mathbf{b}_{i}$ respectively here. Notice that, if we denote the symmetric $3 \times 3$ matrix,

$$
X_{i}=\frac{1}{\left|\mathbf{b}_{i}-\mathbf{a}_{i}\right|^{2}}\left(\mathbf{b}_{i}-\mathbf{a}_{i}\right)\left(\mathbf{b}_{i}-\mathbf{a}_{i}\right)^{T}
$$

then the stiffness matrix can be written as,

$$
K_{i}=k\left(\begin{array}{cc}
A_{i} X_{i} B_{i}^{T} & A_{i} X_{i} \\
X_{i} B_{i}^{T} & X_{i}
\end{array}\right)
$$

where $A_{i}$ and $B_{i}$ are the anti-symmetric matrices corresponding to $\mathbf{a}_{i}$ and $\mathbf{b}_{i}$.

Assuming that the stiffness constants $k$ are the same for all six legs, the overall stiffness matrix of the system is simply,

$$
K=\left(\begin{array}{cc}
\Xi & \Gamma \\
\Gamma^{T} & \Upsilon
\end{array}\right)
$$




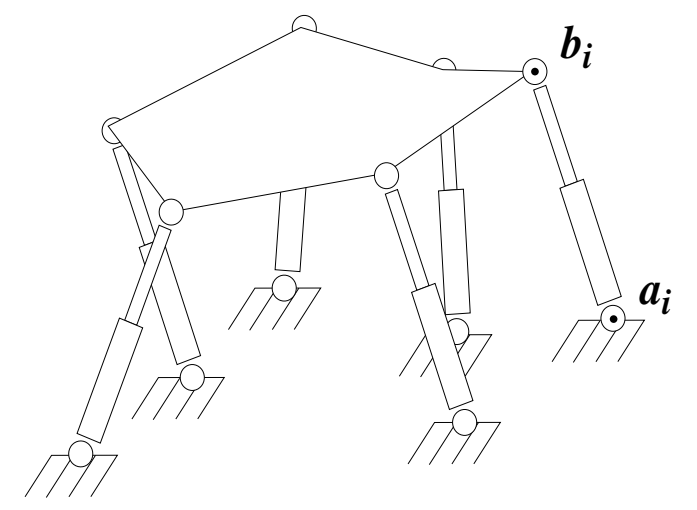

Figure 1: A General Stewart Platform

where,

$$
\begin{aligned}
\Xi & =k \sum_{i} A_{i} X_{i} B_{i}^{T} \quad \Gamma=k \sum_{i} A_{i} X_{i} \\
\Gamma^{T} & =k \sum_{i} X_{i} B_{i}^{T} \quad \Upsilon=k \sum_{i} X_{i}
\end{aligned}
$$

\section{Damping}

The system we are considering is heavily damped so we cannot ignore damping effects. However, we will assume a very simple model for the damping, we assume that all the damping is due to viscous friction in the hydraulic actuator. Each leg will produce a damping wrench and the total damping wrench will be the sum of these wrenches. In each leg the frictional force will be directed along the line joining the centres of the passive joints at the ends of the leg and will be proportional to the rate at which the leg is extending or contracting. So in terms of the velocity of the point $\mathbf{b}_{i}$ the damping force is,

$$
\mathbf{F}_{i}=\frac{\delta}{\left|\mathbf{b}_{i}-\mathbf{a}_{i}\right|^{2}}\left(\mathbf{b}_{i}-\mathbf{a}_{i}\right)\left(\mathbf{b}_{i}-\mathbf{a}_{i}\right)^{T}\left(\boldsymbol{\omega} \times \mathbf{b}_{i}+\mathbf{v}\right)
$$

where $\delta$ is a constant. Rearranging the triple product gives,

$$
\mathbf{F}_{i}=\frac{\delta}{\left|\mathbf{b}_{i}-\mathbf{a}_{i}\right|^{2}}\left(\mathbf{b}_{i}-\mathbf{a}_{i}\right)\left(\left(\mathbf{a}_{i} \times \mathbf{b}_{i}\right)^{T} \boldsymbol{\omega}+\left(\mathbf{b}_{i}-\mathbf{a}_{i}\right)^{T} \mathbf{v}\right)
$$

To find the moment of this force we take the vector product with a point on the line, $\mathbf{a}_{i}$ say,

$$
\mathbf{M}_{i}=\frac{\delta}{\left|\mathbf{b}_{i}-\mathbf{a}_{i}\right|^{2}}\left(\mathbf{a}_{i} \times \mathbf{b}_{i}\right)\left(\left(\mathbf{a}_{i} \times \mathbf{b}_{i}\right)^{T} \boldsymbol{\omega}+\left(\mathbf{b}_{i}-\mathbf{a}_{i}\right)^{T} \mathbf{v}\right)
$$

Hence the damping wrench can be represented by a damping matrix,

$$
\left(\begin{array}{c}
\mathbf{M}_{i} \\
\mathbf{F}_{i}
\end{array}\right)=\delta\left(\begin{array}{cc}
A_{i} X_{i} B_{i}^{T} & A_{i} X_{i} \\
X_{i} B_{i}^{T} & X_{i}
\end{array}\right)\left(\begin{array}{l}
\boldsymbol{\omega} \\
\mathbf{v}
\end{array}\right)
$$

If the damping constant $\delta$ is the same for each leg then it is easy to see that the total damping matrix $D$, is proportional to the stiffness matrix $K$, of the system.

\section{Equations of Motion}

When we put together the results of the last few sections we get the following system of second order linear differential equations,

$$
N \ddot{\mathbf{z}}+D \dot{\mathbf{z}}+K \mathbf{z}=\mathbf{0} .
$$

This equation describes the dynamics of small vibrations about a stationary configuration of the manipulator. In general, such a system is solved by solutions of the form,

$$
\mathbf{z}=\exp \left(\lambda_{j} t\right) \mathbf{z}_{j}
$$


where $\mathbf{z}_{j}$ is a constant screw, substituting this into the equation above gives,

$$
\exp \left(\lambda_{j} t\right)\left(\lambda_{j}^{2} N+\lambda_{j} D+K\right) \mathbf{z}_{j}=\mathbf{0} .
$$

That is, $\lambda_{j}$ is a solution of the characteristic equation,

$$
\operatorname{det}\left(\lambda_{j}^{2} N+\lambda_{j} D+K\right)=0
$$

and $\mathbf{z}_{j}$ is the corresponding generalised eigenvector. Since the symmetric matrices $N, D$ and $K$ have order $6 \times 6$ we expect 12 independent solutions in general.

This problem can be put in the form of a standard eigenvalue problem as follows, consider the 12 dimensional vector, $\mathbf{q}^{T}=\left(\dot{\mathbf{z}}^{T}, \mathbf{z}^{T}\right)$. Now the original equation is equivalent to the 12 dimensional first order system,

$$
\left(\begin{array}{cc}
0 & N \\
N & D
\end{array}\right) \dot{\mathbf{q}}+\left(\begin{array}{cc}
-N & 0 \\
0 & K
\end{array}\right) \mathbf{q}=\mathbf{0}
$$

So again we see that 12 solutions are to be expected. This form however, provides 'orthogonality-like' condition between different solutions. For two solutions with different eigenvalues $\lambda_{j}$ and $\lambda_{k}$ we have,

$$
\left(\lambda_{j}+\lambda_{k}\right) \mathbf{z}_{j}^{T} N \mathbf{z}_{k}+\mathbf{z}_{j}^{T} D \mathbf{z}_{k}=0
$$

and

$$
\mathbf{z}_{j}^{T} K \mathbf{z}_{k}-\lambda_{j} \lambda_{k} \mathbf{z}_{j}^{T} N \mathbf{z}_{k}=0
$$

Eliminating the term containing the inertia matrix we also have that,

$$
\left(\lambda_{j}+\lambda_{k}\right) \mathbf{z}_{j}^{T} K \mathbf{z}_{k}+\lambda_{j} \lambda_{k} \mathbf{z}_{j}^{T} D \mathbf{z}_{k}=0
$$

For our case we have that $D=\xi K$ for some constant $\xi=$ $\delta / k$ and hence this last relation becomes,

$$
\left(\lambda_{j}+\xi \lambda_{j} \lambda_{k}+\lambda_{k}\right) \mathbf{z}_{j}^{T} K \mathbf{z}_{k}=0
$$

and hence either $\mathbf{z}_{j}^{T} K \mathbf{z}_{k}=0$ or $\left(\lambda_{j}+\xi \lambda_{j} \lambda_{k}+\lambda_{k}\right)=0$.

\section{Harmonic Screws}

In his famous treatise [1] Ball described what he called harmonic screws. These were essentially solutions to the dynamical problem,

$$
N \ddot{\mathbf{z}}+K \mathbf{z}=\mathbf{0}
$$

for an arbitrary rigid body sitting at an equilibrium position of an arbitrary potential function. Ball showed that in general there were six harmonic screws and that modal solutions to the equation were oscillations about about these screws. That is,

$$
\mathbf{z}(t)=(\alpha \cos \mu t+\beta \sin \mu t) \mathbf{z}
$$

where the constants $\alpha$ and $\beta$ are determined by the initial conditions. Notice that the oscillation frequency $\mu$ satisfies the characteristic equation,

$$
\operatorname{det}\left(K-\mu^{2} N\right)=0 \text {. }
$$

Because in our problem the damping matrix is proportional to the stiffness matrix our problem reduces to the one above. Assuming as above that $D=\xi K$, the characteristic equation reduces to,

$$
\operatorname{det}\left(\lambda^{2} N+(1+\xi \lambda) K\right)=0
$$

So if we let $-\mu^{2}=\lambda^{2} /(1+\xi \lambda)$ we can write the solutions to the damped problem in term of the undamped frequencies and harmonic screws. The eigenvalues are,

$$
\lambda=-\frac{\mu^{2} \xi}{2} \pm \frac{\mu}{2} \sqrt{\mu^{2} \xi^{2}-4} .
$$

Notice that we only get six eigenscrews but each one appears in two solutions. Suppose we label the two solutions for one harmonic screw $\lambda_{+}$and $\lambda_{-}$, now from the above we have that $\lambda_{+} \lambda_{-}=\mu^{2}$ and $\lambda_{+}+\lambda_{-}=-\mu^{2} \xi$. Multiplying the first of these equations by $\xi$ and adding we get,

$$
\lambda_{+}+\xi \lambda_{+} \lambda_{-}+\lambda_{-}=0
$$

in agreement with the results of the previous section.

\section{Over Damping}

In general, it is desirable that the platform should not vibrate. From the results of the previous section this means that the eigenvalues must be all real. Examining the discriminant we see that we must have,

$$
\mu^{2} \xi^{2}>4
$$

where $\mu$ is the lowest undamped frequency. 
We can find bounds on the values of the frequencies using a slight variation of the Rayleigh quotient, see [4]. Consider the function,

$$
\rho(\mathbf{s})=\frac{\mathbf{s}^{T} K \mathbf{s}}{\mathbf{s}^{T} N \mathbf{s}} .
$$

If $N$ is positive definite, which is usual in dynamics problems, then this function has a maximum which is the square of the largest frequency and a minimum equal to the square of the lowest frequency of the system. To see this assume that the harmonic screws are, $\mathbf{s}_{1}, \mathbf{s}_{2}, \ldots, \mathbf{s}_{6}$. That is, $K \mathbf{s}_{i}=$ $\mu_{i}^{2} N \mathbf{s}_{i}$ for $i=1, \ldots, 6$. It is also useful to assume that the frequencies are ordered so that, $\mu_{1}^{2}>\mu_{2}^{2}>\cdots>\mu_{6}^{2}$. The harmonic screws will be linearly independent so we can write any screw as a linear combination of the harmonic screws,

$$
\mathbf{s}=\alpha_{1} \mathbf{s}_{1}+\alpha_{2} \mathbf{s}_{2}+\cdots+\alpha_{6} \mathbf{s}_{6}
$$

for some constants $\alpha_{i}$. Now the harmonic screws are orthogonal with respect to $N$ and $K$ so that $\mathbf{s}_{i}^{T} N \mathbf{s}_{j}=\mathbf{s}_{i}^{T} K \mathbf{s}_{j}=0$ if $i \neq j$. Substituting this into the definition of the Rayleigh quotient gives,

$$
\rho(\mathbf{s})=\frac{\sum_{i=1}^{6} \alpha_{i}^{2} \mu_{i}^{2} \mathbf{s}_{i}^{T} N \mathbf{s}_{i}}{\sum_{i=1}^{6} \alpha_{i}^{2} \mathbf{s}_{i}^{T} N \mathbf{s}_{i}}
$$

If we take $\rho(\mathbf{s})-\mu_{1}^{2}$ we get,

$$
\rho(\mathbf{s})-\mu_{1}^{2}=\frac{\sum_{i=2}^{6} \alpha_{i}^{2}\left(\mu_{i}^{2}-\mu_{1}^{2}\right) \mathbf{s}_{i}^{T} N \mathbf{s}_{i}}{\sum_{i=1}^{6} \alpha_{i}^{2} \mathbf{s}_{i}^{T} N \mathbf{s}_{i}}
$$

which is clearly negative for any $\mathbf{s}$. That is, $\rho(\mathbf{s}) \leq \mu_{1}^{2}$, equality being achieved when $\mathbf{s}=\mathbf{s}_{1}$. On the other hand we have that,

$$
\rho(\mathbf{s})-\mu_{6}^{2}=\frac{\sum_{i=1}^{5} \alpha_{i}^{2}\left(\mu_{i}^{2}-\mu_{6}^{2}\right) \mathbf{s}_{i}^{T} N \mathbf{s}_{i}}{\sum_{i=1}^{6} \alpha_{i}^{2} \mathbf{s}_{i}^{T} N \mathbf{s}_{i}}
$$

which is obviously positive and hence, $\mu_{6}^{2} \leq \rho(\mathbf{s})$ with equality when $\mathbf{s}=\mathbf{s}_{6}$. The maximum and minimum values of this function are the squares of the highest and lowest undamped frequencies of the system. The rest of the frequencies turn up as other stationary values of the function.

Hence for a fully damped system we must have,

$$
\xi^{2} \rho(\mathbf{s})>4,
$$

for all screws s. Another way of putting this is to write,

$$
\xi^{2} \mathbf{s}^{T} K \mathbf{s}-4 \mathbf{s}^{T} N \mathbf{s}>0
$$

so that the condition becomes that the symmetric matrix $\left(\xi^{2} K-4 N\right)$ is positive definite.

The standard way to determine if a symmetric matrix is positive definite is to check that all it's leading minors are positive, see [2, sect. 8.2] for example. Notice however, that most of the leading minors are not coordinate invariant. Of course, their signs are invariant with respect to coordinate transformations.

Another characterisation of $6 \times 6$ positive definite symmetric matrices is due to Patterson and Lipkin [5]. This involves a slightly different eigenvalue problem. The eigenstiffnesses of a $6 \times 6$ symmetric matrix are the solutions $\gamma$, to the eigenvalue problem,

$$
M \mathbf{s}=\gamma Q_{0} \mathbf{s}
$$

where $Q_{0}$ is the matrix $\left(\begin{array}{cc}0 & I_{3} \\ I_{3} & 0\end{array}\right)$. These ideas date back to Ball [1] who also called the eigenscrews s principal screws. The contribution of Patterson and Lipkin was to show that the matrix $M$ was positive definite if and only if it has three positive and three negative eigenstiffnesses. They also showed that the principal screws must have pitches with the same sign as their corresponding eigenstiffnesses. In fact, if we let $P=\left(\mathbf{s}_{1}\left|\mathbf{s}_{2}\right| \cdots \mid \mathbf{s}_{6}\right)$ be the matrix whose columns are given be the principal screws, then it is not hard to see that this matrix diagonalises the original matrix $M$. Recall, that the principal screws are mutually reciprocal; $\mathbf{s}_{i}^{T} Q_{0} \mathbf{s}_{j}=0$ if $i \neq j$. Moreover, the diagonal entries are given by $\gamma_{i} \mathbf{s}_{i}^{T} Q_{0} \mathbf{s}_{i}$ and are thus all positive.

So our condition for no oscillations becomes,

$$
\mathbf{s}_{i}^{T} K \mathbf{s}_{i}>\frac{4}{\xi^{2}} \mathbf{s}_{i}^{T} N \mathbf{s}_{i}, \quad i=1, \ldots, 6
$$

or using the result we have for the form of the stiffness matrix,

$$
\sum_{j=1}^{6}\left(\mathbf{w}_{j}^{T} \mathbf{s}_{i}\right)^{2}>\frac{4 k}{\delta^{2}} \mathbf{s}_{i}^{T} N \mathbf{s}_{i}, \quad i=1, \ldots, 6
$$

where $\mathbf{w}_{i}$ if the unit wrench along the $i$ th leg.

\section{Conclusions}

The key question now is whether or not the symmetric matrix $\left(\xi^{2} K-4 N\right)$ can be positive definite over the whole 
work-space of the Stewart platform? Unfortunately this question seems to be difficult to answer in general. However, given the parameters of a particular machine it should not be too difficult to compute. Remember here that as the platform moves the inertia matrix as well as the stiffness matrix will change. However, if we are only interested in whether or not the combination $\left(\xi^{2} K-4 N\right)$ is positive definite then we may choose any coordinate frame. For example we could choose a frame with origin at the platform's centre of mass and with axes aligned with the principal axes of the platform. This makes $N$ diagonal and the changes in $K$ only arise from the movement of the base points $\mathbf{a}_{i}$.

The model presented here for the vibrations of Stewart platforms has been very simple but is it too simple to be realistic? There are several assumptions we have made which may not be completely accurate, for example we assumed that the damping and stiffness constants were the same for each leg. Assuming small manufacturing tolerances for the hydraulic actuators this is probably reasonable. But are these constants the same whatever the extension of the leg?

Another effect we have ignored is friction in the passive spherical joints. It does not seem to be too difficult to include a simple model of viscous friction ta these joints, we could assume a torque resisting the rotation of the joint proportional to the angular velocity of the joint. It might even be possible to derive a damping matrix for such a model. However, in such a model the damping matrix would not be proportional to the stiffness matrix and the analysis would be substantially more difficult.

Although we have only looked at the quasi-static case here, where the actuators are not driven, it should be possible to extend this work to the case the platform is moving along some nominal trajectory. The goal here would be to derive the linearised dynamics about the given trajectory. If we include a force on the platform then this would be directly applicable to hexapod machine-tools.

Finally, in this work hydraulic actuators have been referred to throughout. That has been for convenience only. The theory applies to other types of actuators, in particular pneumatic actuators, which of course would be more compliant.

\section{References}

[1] R.S. Ball. The Theory of Screws. Cambridge University Press, Cambridge, 1900.

[2] P.M. Cohn. Algebra, volume 1. John Wiley and Sons, London, 1974.

[3] S. Huang and J.M. Schimmels. The bounds and realization of spatial stiffnesses achieved with simple springs connected in parallel. IEEE Trans. on Robotics and Automation, 14(3):466-475, 1998.

[4] B. Noble. Applied Linear Algebra. Prentice Hall, New Jersey, 1969.

[5] T. Patterson and H. Lipkin. Structure of Robot Compliance. J. Mechanical Design 1993 vol. 115 pp. 576-580.

[6] J.M. Selig. Geometrical Methods in Robotics. Springer Verlag, New York, 1996. 